\title{
Research on Network System of Smart Substation Typical Problems and Its Corresponding Solutions
}

\author{
Xiaofei Zhang ${ }^{\mathrm{a}}{ }^{*}$ Luolin Zheng $^{\mathrm{b}}$ and Ruying Zhao ${ }^{\mathrm{c}}$ \\ State Grid Electric Power Research Institute, Testing and Verification Center, Nanjing, China \\ azhangxiaofei@sgepri.sgcc.com.cn, \\ bzhengluolin@sgepri.sgcc.com.cn, \\ czhaoruying@sgepri.sgcc.com.cn
}

\begin{abstract}
Key words: smart substation, network structure, IEC 61850, intelligent electronic device Abstract: The structure of the intelligent substation network is analyzed. The typical design is discussed for the process level network, the station control layer network and the time synchronization network. On this basis, the typical issues of the intelligent substation network are analyzed and corresponding solutions are proposed.
\end{abstract}

\section{Introduction}

With the development of primary equipment such as unconventional mutual inductor and intelligent switch, together with the continuous renewal of network communication technology, especially the wide use of IEC61850-based substation network and communication protocol standard, the information collection, transmission, processing, and output of intelligent substations have had unified data model and data communication platform. Thus total stations have achieved digitalized information, communication platform network, standardized information sharing, and automated operation and management [1].

Intelligent substations have improved many deficiencies of the conventional substations in terms of information interactions. During the information interactions of intelligent substations, the electromagnetic interference of hardwire secondary cable is reduced. As a result, the information could be shared, and the operation and management efficiency has been improved. IEC61850 enables the equipment's interoperability and secondary device's unified interface specification [2]. However, there are several aspects for improvement in intelligent substation network's actual operation, which is constructed with the power system architecture and information system. This article primarily addresses these topics.

\section{Typical Network Structure Design for Intelligent Substations}

Intelligent substations use DL/T860 (IEC61850) as the internal communication and modeling standard. Based on the methodology of "separation of communication service and communication realization", the main communication business of substations includes: Sampled Measured Values (SMV), Generic Object Oriented Substation Events (GOOSE), Pairing services, and Infrastructure Services (core ACSI services) [3]. With the emergence of advanced applications such as fault recorder, network analysis system, and video system, different service varies in the requirement for network resource [4]. IEC61850 proposed the three-tier-two-network framework for intelligent substation automation system. By studying the division and functionality of the framework, the form of architecture for different construction stages, and further the overall demand of the substation network, can be determined.

Process-level network. At the beginning of the intelligent substation construction, in order to ensure the reliability of the process layer data, point-to-pinto model is adopted in digital 
substation's process layer connection in addition to dual protection and control. The protection device and merging unit are directly connected, current and voltage data are transmitted via direct wire, and protective tripping and reclosing command are also transmitted to intelligent terminals through wired connection. The measurement device's remote measurement values and commands are processed via process layer exchange acidity unit network. The typical network structure of point-to-point model is shown in Figure 1.

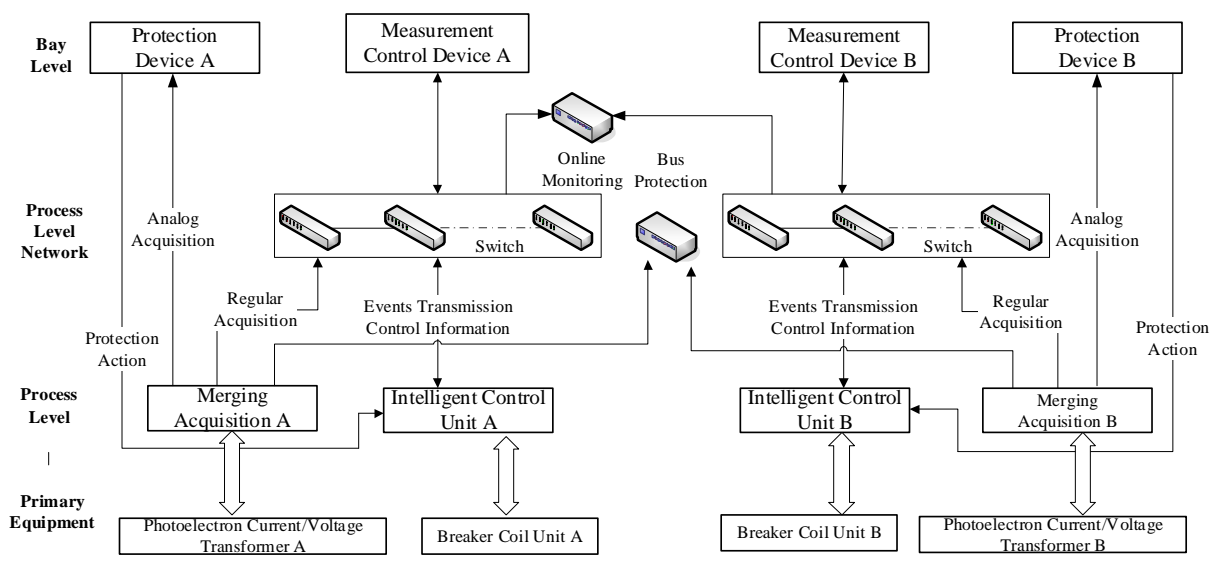

Fig. 1 Network structure of point-to-point model

Protection device and measurement device are wired connected to merging unit. Therefore, current and voltage data can be sent directly, and the commands from tripping and reclosing protection and measuring and control are transferred to the process layer intelligent operation box through GOOSE. Dual measurement protection is also configured. The structure of point-to-point gathering and GOOSE trip network is shown in Figure 2.

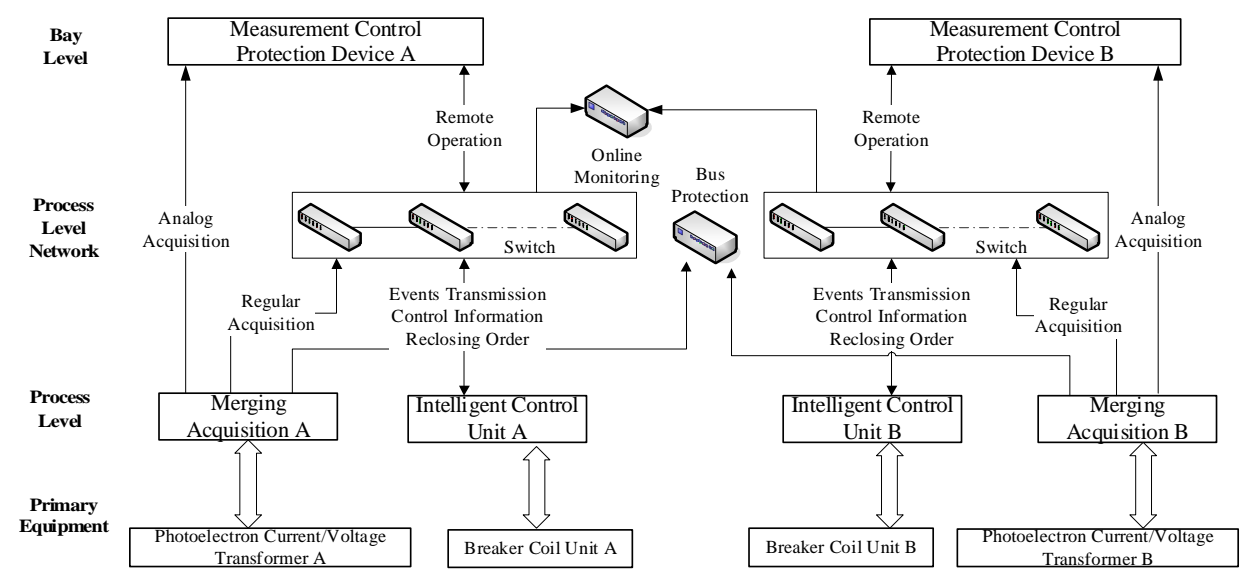

Fig. 2 Network structure of point-to-point gather and GOOSE trip network

In such networking mode, protection measurement and control both adopt the dual configuration. Remote measurement collection like current, voltage and phase, as well as commands such as protection tripping, reclosing, and remote control are forwarded through process layer switches. SV network in process layer and GOOSE network are physically isolated. Through the SV network, the current and voltage data of merging unit are forwarded to upper measurement and protection device; While commands like protection tripping reclosing and remote control are forwarded for execution to process layer intelligent operation box through GOOSE. The typical network structure of separated remote measurement gathering and GOOSE trip are shown in figure 3. 


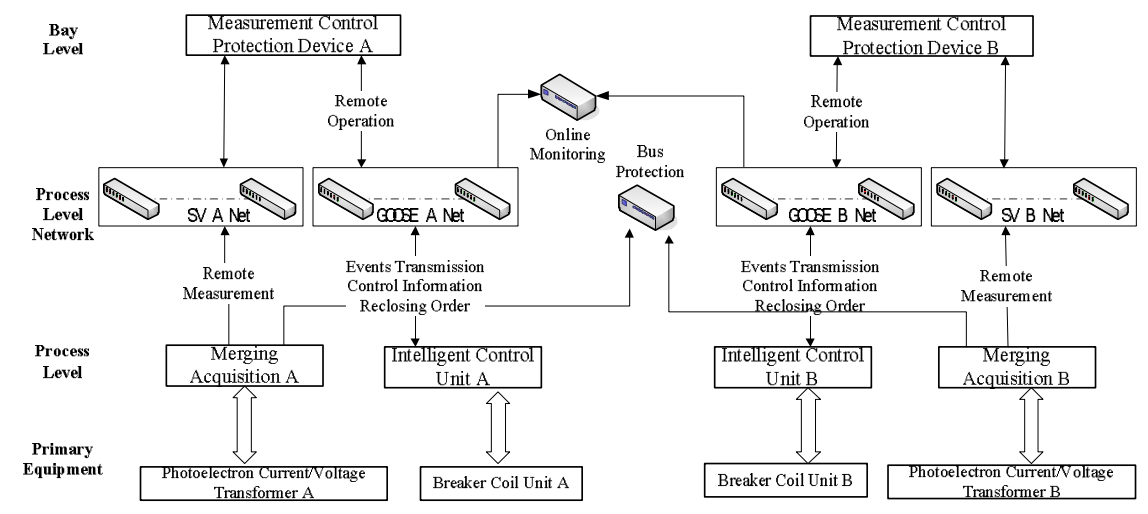

Fig. 3 Separated network structure of remote measurement gather and GOOSE trip

By merging process layer SV network and GOOSE network into one physical network, remote measurement collection like current, voltage and phase, as well as commands such as protection tripping, reclosing, and remote control are all forwarded through process layer switches. Through the process layer network, the current and voltage data of merging unit are forwarded to upper measurement and protection device; While commands like protection tripping reclosing and remote control are forwarded for execution to process layer intelligent operation box, different functional data flows are logically separated by switch VLAN. Thus the SV network and GOOSE network are integrated. The typical dual network structure is shown in figure 4.

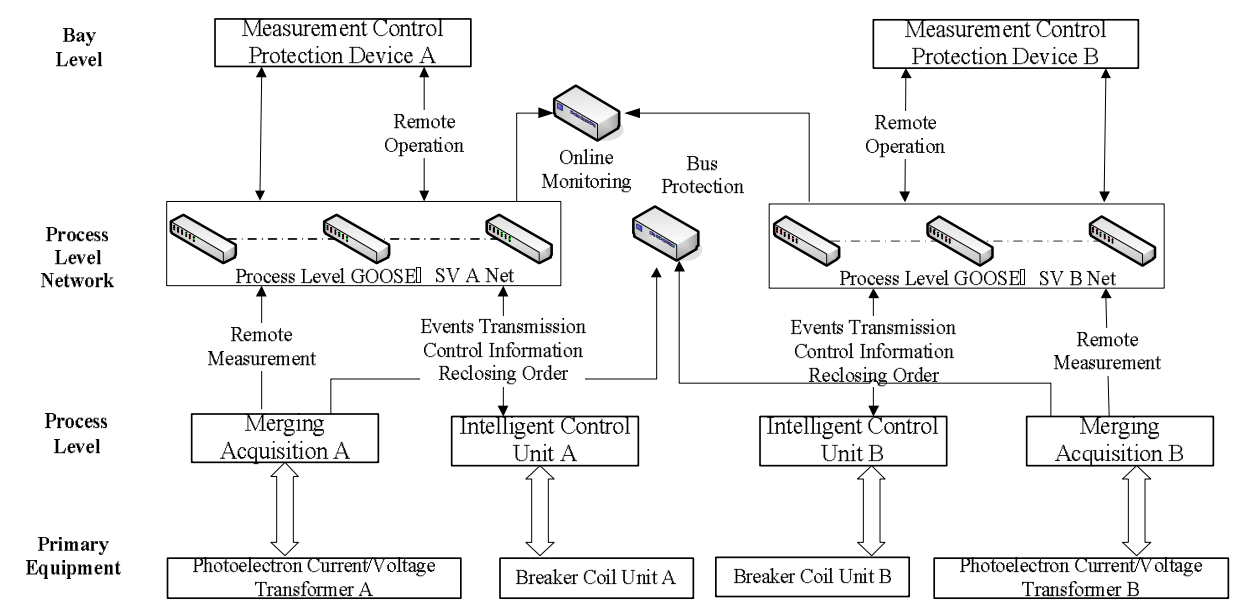

Fig. 4 Network structure of Dual-network integration

The characteristics of each network structure of process layer are shown in table 1 . 
Table1 Network structure characteristics of process layer

\begin{tabular}{|c|c|c|}
\hline $\begin{array}{l}\text { Network } \\
\text { Structure }\end{array}$ & Pros & Cons \\
\hline $\begin{array}{l}\text { point-to-point } \\
\text { model }\end{array}$ & $\begin{array}{l}\text { Improved } \\
\text { protection } \\
\text { reliability }\end{array}$ & $\begin{array}{l}\text { Large number of cables; more } \\
\text { fiber for processing layer / } \\
\text { protection devices; high calorific } \\
\text { value, hard to maintain }\end{array}$ \\
\hline $\begin{array}{l}\text { point-to-point } \\
\text { gather and } \\
\text { GOOSE trip } \\
\text { network }\end{array}$ & $\begin{array}{l}\text { Secondary } \\
\text { equipment } \\
\text { network, } \\
\text { reduced cables }\end{array}$ & $\begin{array}{c}\text { Direct SV, complex network } \\
\text { structure }\end{array}$ \\
\hline $\begin{array}{l}\text { remote } \\
\text { measurement } \\
\text { gather and } \\
\text { GOOSE trip } \\
\text { separation }\end{array}$ & $\begin{array}{l}\text { Processing layer } \\
\text { network, less } \\
\text { cable }\end{array}$ & $\begin{array}{l}\text { SV/GOOSE separation, more } \\
\text { processing layer switch, complex } \\
\text { network structure }\end{array}$ \\
\hline $\begin{array}{l}\text { Dual-network } \\
\text { integration }\end{array}$ & $\begin{array}{l}\text { Processing layer } \\
\text { network, less } \\
\text { cable and } \\
\text { switches }\end{array}$ & $\begin{array}{l}\text { Large data transmission load; } \\
\text { SV/GOOSE data logical } \\
\text { separation; high performance and } \\
\text { security requirement for } \\
\text { switches/devices }\end{array}$ \\
\hline
\end{tabular}

Substation control layer network. For communication protocols, equipment from each manufacturer communicates according to the MMS in IEC61850 communication standard protocol. Substation control layer uses unicast messaging to forward data stream based on the address learning function of industrial Ethernet switches, which is different from the GOOSE / SV double group messaging in process layer. Thus from the perspective of networking mode, there isn't much difference between intelligent substation's control layer network structure and the Ethernet transmission based conventional integrated automation substation. Substation control layer network and process layer network are separated into two independent networks by interval layer isolation equipment. Substation control layer mostly adopts double stranded wire interconnection, while process layer multimode fiber connection. Intelligent equipment such as measurement and control and protection share data through switches, substation layer equipment directly acquires data such as current, voltage, phase angle, frequency, power, gear, oil temperature, switch position, remote communication condition from the station control switch. In addition, network analyzer and other advanced applications for message storage and analysis are added to substation layer, providing data analysis functionality in case of malfunctions. The typical network structure of substation control layer is shown in figure 5.

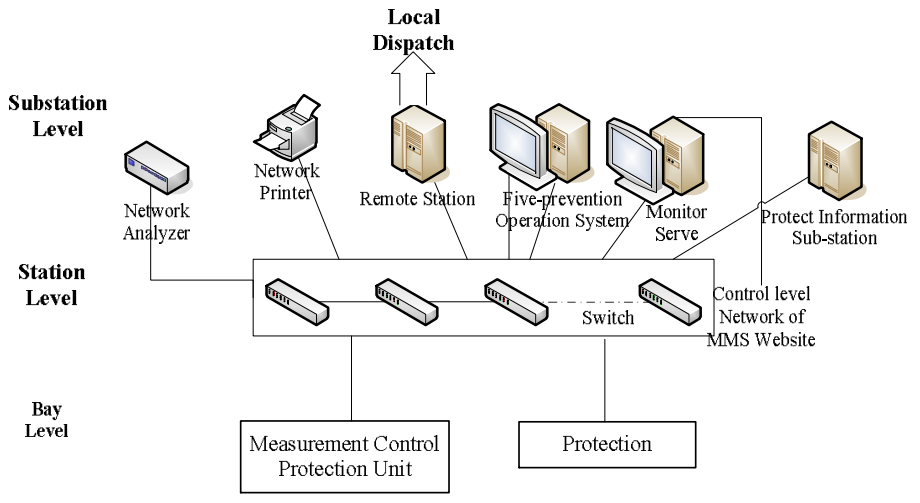

Fig. 5 Typical network structure of station control layer 
Time synchronization network. Due to the fact that time is the benchmark of current and voltage data uploaded to interval layer protection devices from process layer so as to prevent abnormality such as differential current, intelligent substations are stricter to timing compared to conventional stations. Currently, there are primarily 4 ways to design the intelligent substations time synchronization network.

Electric B code synchronization should be assisted with network synchronization that cannot work as the main measure alone for a long time. The prompt recovery of code B synchronization should be ensured. Due to RS485's distortion when going through high-pressure field, time synchronization system is less used in intelligent stations.

Process layer merging unit adopts optical B code synchronization to avoid high-pressure disturbance. Most equipment of interval layer and substation layer are located in chambers with good condition, when electric B code synchronization fails, network synchronization will be hot standby. When process layer merging unit use to optical B code synchronization directly, dual master clocks as hot main case are required to ensure the reliability of time synchronization.

When optical B code synchronization is used in substation layer, interval layer and process layer, dual master clocks are required as hot main case. A typical topology of combination of optical code B and network synchronization is shown in figure 6.

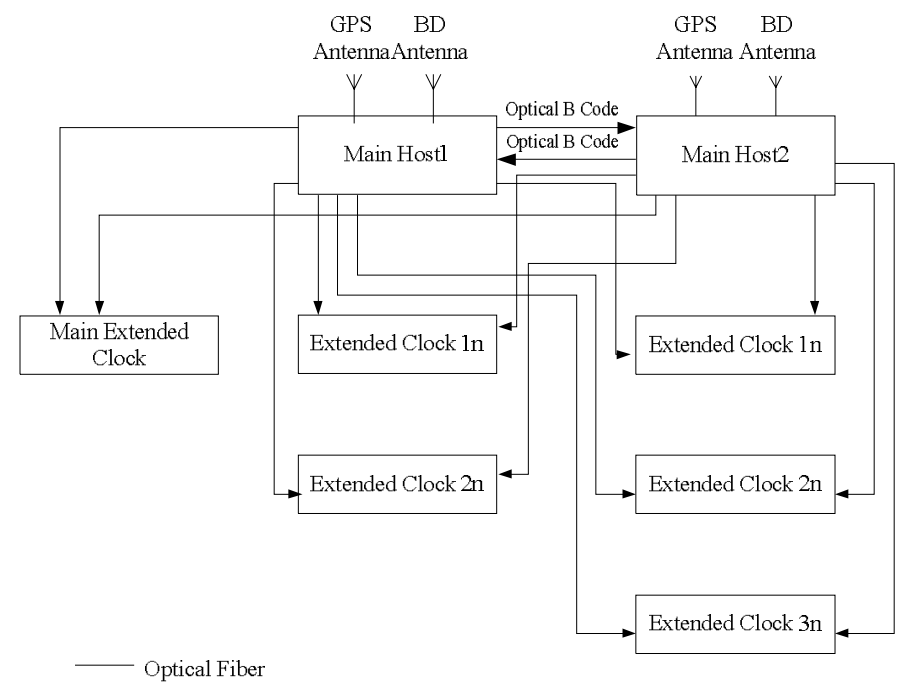

Fig. 6 Optical B code synchronization and network synchronization combination

IEEE1588 is a time synchronization methodology with high accuracy and reliability, and has high technical requirements for secondary equipment, which includes master-slave dual clocks setting, time scale for secondary equipment message, and switches supporting 1588 clock synchronization [6]. Although few intelligent substations have adopted the IEEE1588 time synchronization so far (such as YanAn 330kV smart station), with the advancement of intelligent station construction, IEEE1588-based synchronization will become the mainstream. The typical typology of IEEE1588 synchronization is shown in figure 7. 


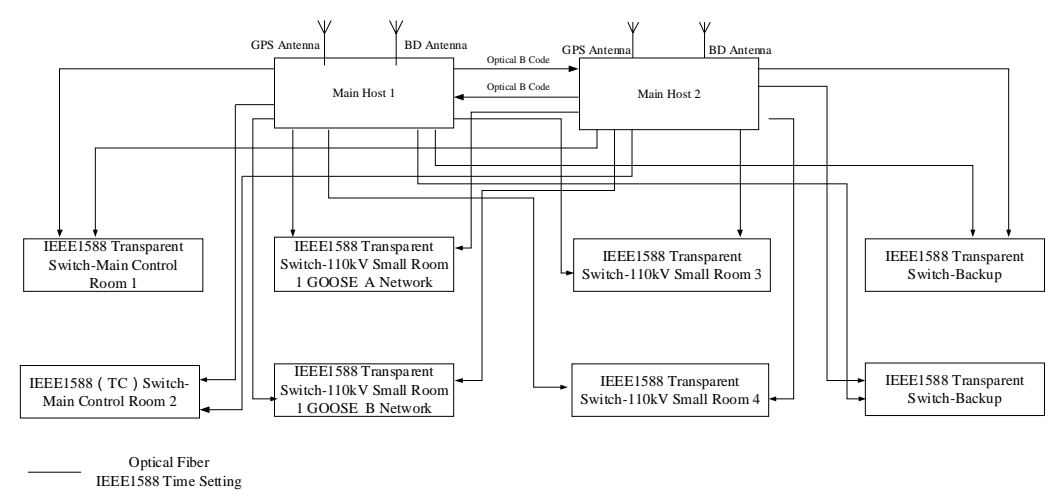

Fig. 7 Typical network of IEEE 1588 synchronization

\section{Typical problems analysis and countermeasures for intelligent stations network}

MMS network mirror does not meet the demand. Power system dedicated industrial Ethernet switches can achieve "Multiple-to-One" mirror image. With a single mirror export, namely single switch can map data streams from multiple ports onto one mirror port, which can be accessed by advanced applications in the smart substation (i.e. packet analysis recorder, online diagnosis system, fault wave record system, etc.) to receive the interacting messages from terminal control layer. Under normal circumstances, there is only one packet analysis recorder as the advanced application of terminal control layer, as shown in figure 8. When there are multiple advanced applications, they will be connected to mirror ports from different switch images.

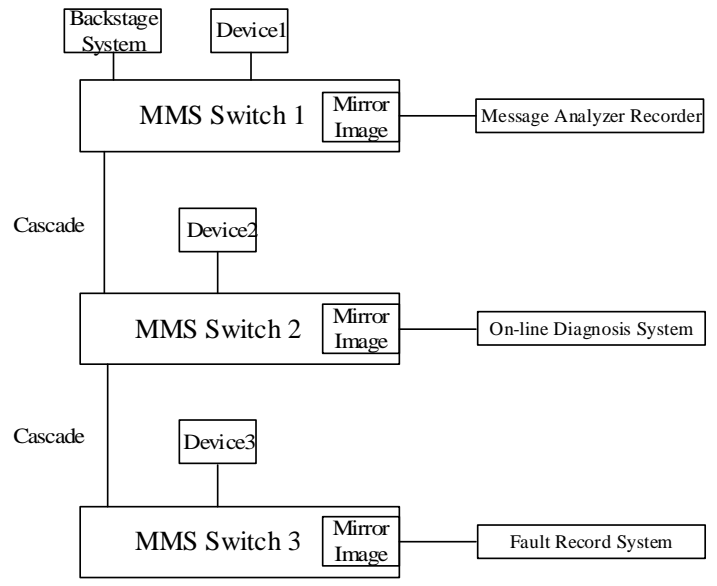

Fig. 8 More advanced applications access

According to the requirements of IEC61850, the MMS message of terminal control layer should be unicast. Unlike GOOSE and SV whose messages are sent in broadcasting, the address learning function of the terminal control layer switches will send MMS message to the corresponding port according to the destination MAC address table. Therefore, part of advanced applications cannot receive the entire device-exchanging messages from MMS layer. As shown in figure 8 , the communication messages from backend system and device 1 cannot be transferred to online diagnosis system and fault wave record system, messages from backend system and devices 2 cannot reach fault wave record system, causing part of the two advanced applications malfunctioning.

In this case, if all three advanced applications are to receive the entire background system messages at the same time, the only solution is through introducing the HUB, and connecting it with the MMS switch 1's mirror port of the backend system, which is shown in figure 9. The MMS messages from these 3 port mirrors can thus be sent to all the advanced applications via the HUB's physical forwarding function. In the substation environment, industrial-level hubs must be configured, or use industrial-level switches with closable address learning function instead. 


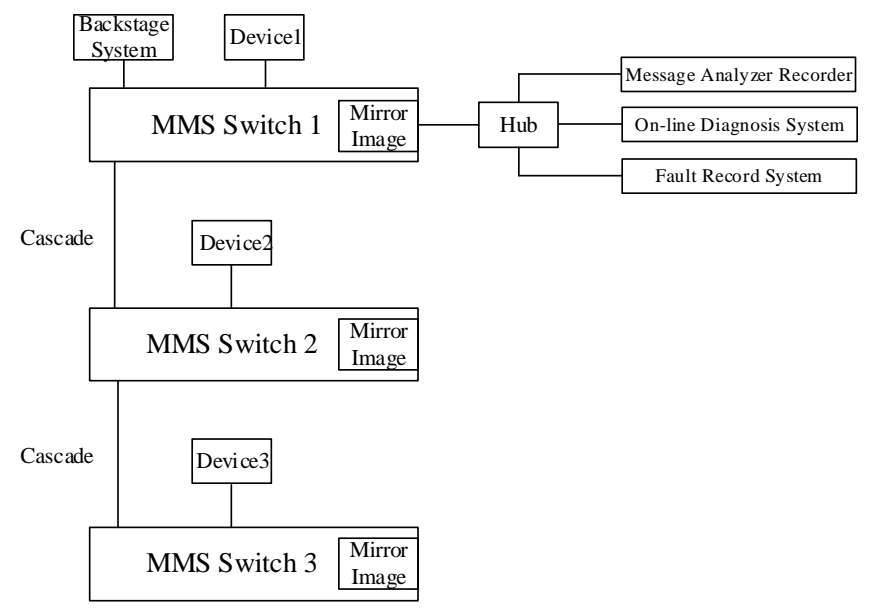

Fig. 9 More advanced applications access Hub

VLAN cannot completely solve the data flow inaccuracy and bandwidth usage. Process layer sends double-layer multicasting messages. In order to avoid devices receiving too much waste packets to cause waste of resources and network bandwidth, the process layer network generally adopt the dividing of VLAN to isolate logic broadcast domain. Ports with subscription relationship will be in the same VLAN, process layer messages are duplicated and exchanged within all ports of a VLAN, and consume switches' physical resources. As shown in figure 10, parts of ports of four switches ABCD are assigned to VLAN1, when IED1 and IED2communicate, $\mathrm{B}$ and $\mathrm{D}$ will alsoreceive the same data, causing data flow inaccuracy and bandwidth waste.

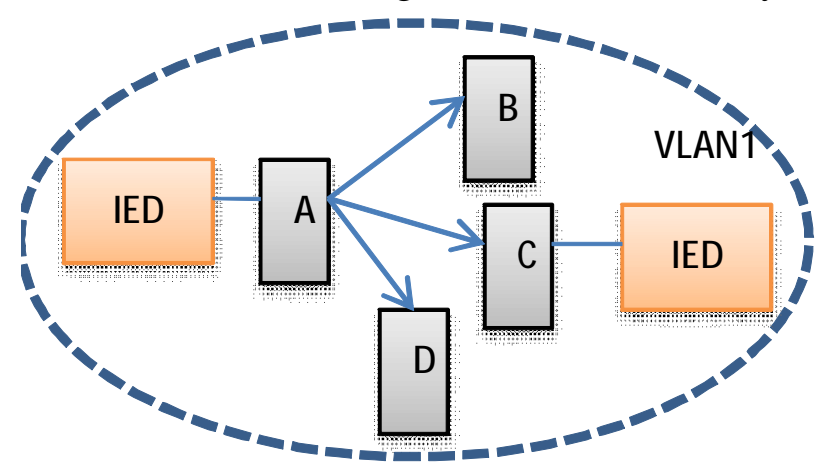

Fig. 10 Port replication in the same VLAN

After the process layer intelligent device receiving a chunk of double-layer broadcasting messages, based off a certain filtering rules, in addition to relying on the MAC address filtering, they also need to check flag bits such as APPID, DADASET, GOID, GOCDREF [7]. Through figure 11, we can see that if the switch can get the subscription relations by parsing SCD file, and form a fixed forwarding table based off flag bits like APPID, message forwarding will be directed, then the problem of data flow inaccuracy and bandwidth consumption can be solved.

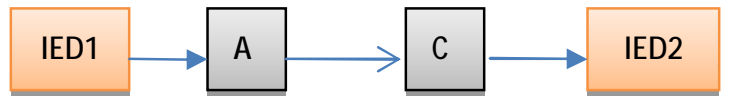

Fig. 11 Clear directivity of data flow

Ring redundancy network causes logical link bridge connect multiple switches. Ring redundancy network is key to increase intelligent station's network fault tolerance. But due to the lack of usage standard, the protocol consistency issues need to be addressed. For example, several private redundancy protocols such as RapidRing, Supreme-Ring, DT-Ring and ESR are used, in order to ensure interactions between switches of various types from different manufacturers after station upgrade or configuration change, usually RSTP and MSTP standard protocols are used. In practice, the logical link disconnection in the ring network will cause the data flow bridge connecting multiple switches. A $750 \mathrm{kV}$ smart

Station's GOOSE topology is shown in figure 12. Due to the low priority of the switch setting, 
the direct link between test switch 1 and 2 is blocked, only when other paths fail will it become an actual transmission channel, which acts as link redundancy backup. It results in the data transmission between test switch 1 and 3 data actually connect to 7 switches in the first and fourth group. "Q/GDW394-2009 330kV-750kV intelligent substation Design Regulations" and "Q/GDW441-2010 intelligent substation relay protection technology standard" both pointed out that "the data transmission route between any two intelligent devices shall not be more than 4 switches". The topology cannot meet this requirement [7-8].

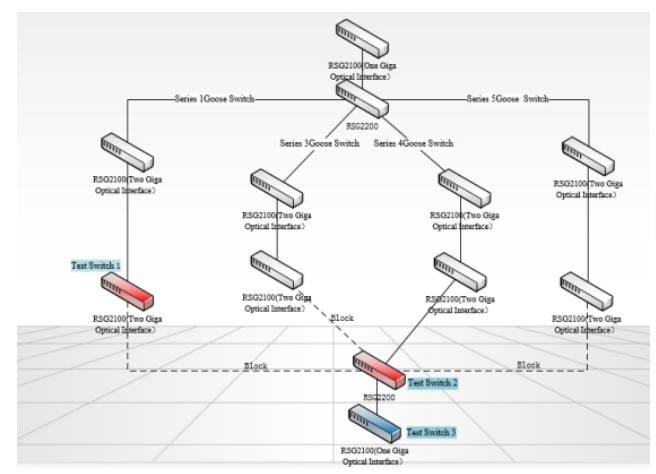

Fig. 12 GOOSE net topology of a $750 \mathrm{kV}$ smart substation

The priority of important messages on the process layer can not be absolutely guaranteed. In order to guarantee the service quality of high-priority packets, the industrial Ethernet switches used by the process layer are usually set as absolute priority. The Ethernet frame format with priority is shown in figure 13 and the recommended value of process layer service is given in table 2 .

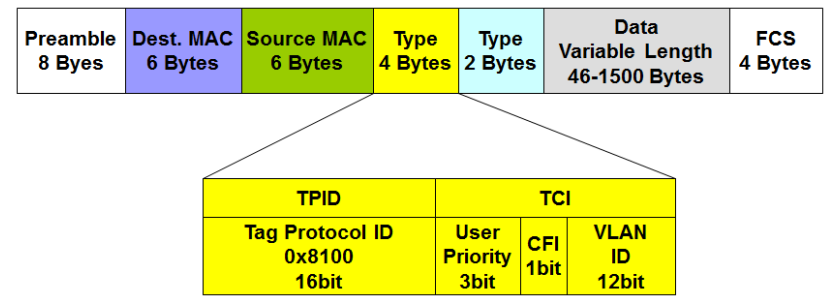

Fig. 13 Ethernet frame format with priority

Table2 Value of IEC61850 recommendations

\begin{tabular}{rcccc}
\hline Service & $\begin{array}{c}\text { Protocol } \\
\text { Type }\end{array}$ & Starting Address & Ending Address & Priority \\
\hline GOOSE & $88 \mathrm{~B} 8$ & 01-0C-CD-01-00-00 & 01-0C-CD-01-01-FF & 4 \\
SMV & 88BA & 01-0C-CD-04-00-00 & 01-0C-CD-04-01-FF & 4 \\
\hline
\end{tabular}

The priority values of GOOSE and SMV packets are both set as 4 usually, with no distinguish among specific business according to the practical experience of intelligent substation project. So process layer switches determine the service quality through the priority value of packets. Packets with same priority still need to queue up in principle of first-in-first-out. Tripping message and other Fast GOOSE with same priority need to wait in line. As shown in figure 14, real time of tripping message may be affected and even lost when the queue is too long.

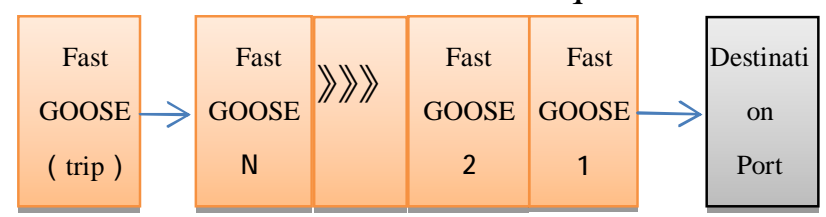

Fig. 14 The same priority queue

It is shown in figure 15 that important messages can be sent with real time and credible in any 
case if the switch can not only identify the priority value, but also prioritize important packets(such as tripping message), leaving specific resources for important messages.

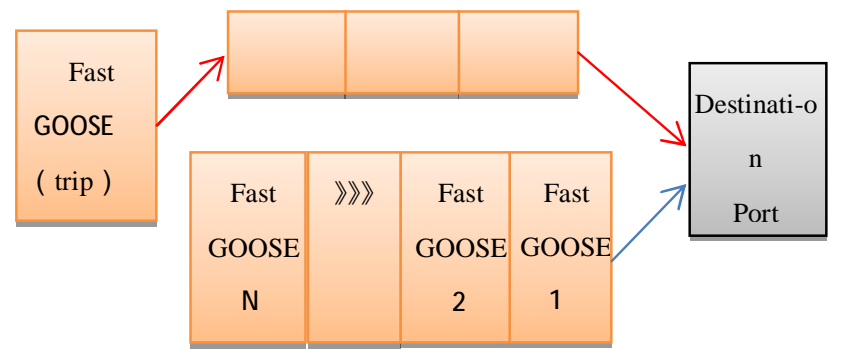

Fig. 15 Important message priority processing

Mixed insertion of IED network port. Mixed insertion of IED network port often happens when debugging and system-level testing the intelligent substation. IEC is inserted to any port of the network and set on the basis, not following the port access design strictly. Although it is easy to debug and test, engineering cabling of the construction site is often designed according to the design drawing strictly. So the network needs to be reset according to the design after laboratory testing, wasting a lot of manpower and material resources.

It is shown in figure 16 that the network configuration just needs one time and the condition of network insertion and debugging are the same which can avoid abnormal conditions caused by different network configuration, making complicated work easy and standard if the switch can load all the SCD files of the station, bond IED and switch bond switch port and give an alarm when it is inserted to the wrong port.

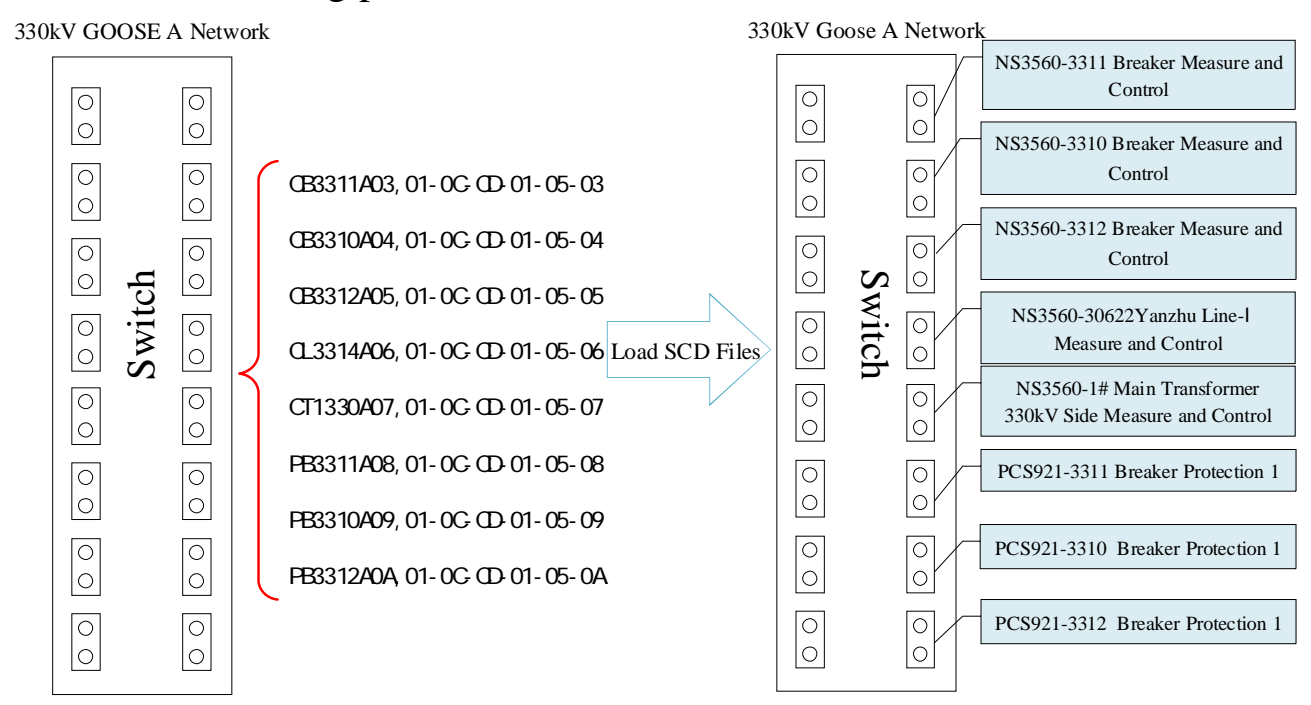

Fig. 16 Network port is automatically bound IED

Transmission with common network causes mutual effect among different messages. The transmission mode based on common network includes GOOSE, SV, MMS, synchronization, broadcasting and the rest. GOOSE and SV messages are divided into fast and common messages. The equipment functions of layer 2 3 and layer $4 \sim 7$ are combined with the complexity of substation layer equipment increases and multiple types of data are included in network channel. The switch has only four priority queues and it is obviously that the need can not be met to distinguish between the resource utilization of messages only with priority. The mutual effect among different messages result from resource competition may cause increasing delay time and packet loss under common network transmission.

As shown in figure 17, the service quality of message transmission can be guaranteed if the switch can divide different messages into logical subnets and allocate independent physical resource to logical subnets to realize mutual isolation. The abnormal situation of certain type message will not affect the transmission of other messages and the stability and reliability of the 
whole intelligent substation can be guaranteed.

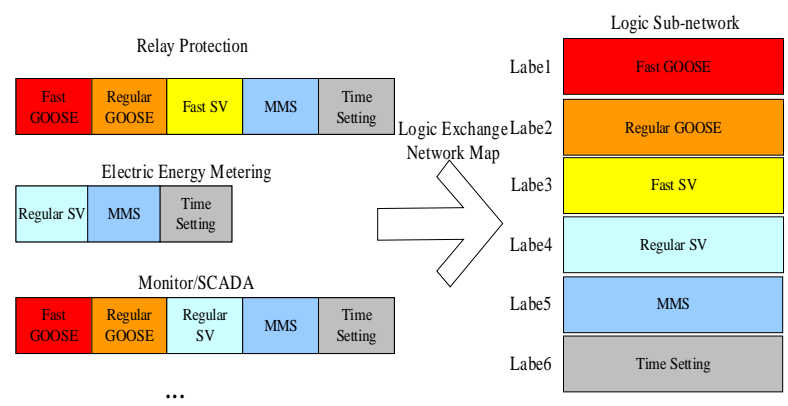

Fig. 17 Logical subnet ensure the message service quality

\section{Conclusions}

Some technical problems in network structure design for intelligent substation have been solved after years of study, construction, verification and discussion. The optimize network solution of the new construction and reconstruction of the substation can be chosen according to the actual condition. Some technical problems met in the construction and operation and maintenance need to be solved during the intelligent process of the substation. In order to solve these problems, the resolving function and performance of application layer should be enhanced with industrial Ethernet switch in addition to the intelligent substation itself. Besides, it is important for the future development of the intelligent substation to take the current situation and future development of the primary and secondary device into consideration.

\section{References}

[1] Xiang Gao, Application prospects of digital substations, J. East China Electric Power. 2006, 34(8):47-53. In Chinese.

[2] Xiaofei Zhang, Peijuan Li, Jiesong Wang, Study on Smart Substation Network Applications and

Testing Technology, J. Jiangsu Electrical Engineering. 2012 , 31(4):34-38. In Chinese.

[3] IEC 61850, Communication Networks and Systems in Substation.2003.

[4] Q/GDW 383-2009 Intelligent Substation Technical Guidance.2009.

[5] Qiangsheng Bu, Yubo Yuan, Xinyue Gong,Tripping Time Characteristics in Smart Substation Process Level Network, J. Electric Power. 2014, (9):137-142. In Chinese.

[6] Xin Huang, Yongfu Wang, Daonong Zhang,IEC 61588 Time Synchronization System and Security Evaluation for SmartSubstations, J. Automation of Electric Power Systems, 2012, 36(13):76-80. In Chinese.

[7] Q/GDW394-2009 330kV-750kV Intelligent substation design specification.2009.

[8] Q/GDW441-2010 Intelligent substation relay protection technology specification.2010.

[9] Xiaofei Zhang, Fuzhong Dan, Ying Ma, Research on networking testing technology of industrial Ethernet switch used in smart substations, J. Telecommunicationsfor Electric Power System, 2010, 31(7):1-4. In Chinese. 